\title{
VARICELLA AND CEREBRAL INFARCTION
}

A relation between varicella and idiopathic arterial ischemic stroke in children was determined in a case control study at the Universite Catholique de Louvain, Brussels, Belgium, and the Centre Hospitalier Bicetre, France. Strokes were confirmed by arteriography in 11 of 41 children, ages 1 month to 15 years (mean, 4 years), referred for arterial infarct, Jan 1985-June 1996. Seven of the 11 children with stroke (64\%) had been diagnosed with varicella within the previous 9 month period (median, 6 weeks; range, 9 days to 9 months) compared to 4 of 44 $(9 \%)$ in the control group. The difference was significant $(\mathrm{p}<0.001)$. The incidence of varicella in the control group was equal to that expected in the general pediatric population in France. A lack of subsequent recurrence of stroke and regression or stabilization of the infarct after at least 18 months of follow-up are supportive of a temporal relationship between varicella and the later development of cerebral infarction. (Sebire G, Meyer L, Chabrier S. Varicella as a risk factor for cerebral infarction in childhood: a case-control study. Ann Neurol May 1999;45:679-680). (Respond: Dr Guillaume Sebire, Service de neurologie, Departement de pediatrie, Cliniques Universitaires Saint Luc, Universite Catholique de Louvain, 10 avenue Hippocrate, 1200 Brussels, Belgium).

COMMENT. Delayed onset hemiparesis and cerebral vascular thrombosis occurring approximately 6 weeks after primary varicella zoster virus infection may explain some cases of idiopathic stroke in children. Bodensteiner JB and associates have previously described the clinical features of vascular thrombosis following varicella (AIDC 1992;146:100-102), and Mintz M, Epstein LG, and Koenigsberger MR found that idiopathic childhood stroke may be associated with human leukocyte antigen (HAL-B51), a common immunogenetic marker, suggesting a genetic predisposition (Ann Neurol 1992;31:675-677). Host factors triggered by viral infection such as varicella may contribute causally to the vascular occlusion. The delay in onset of stroke could result from the time taken for the vascular media to be infected with the virus (see Progress in Pediatric Neurology II, PNB Publishers, 1994;p376 for reports and commentary).

Varicella related childhood stroke occurs in early childhood, is usually delayed for weeks after the rash, and infarcts, best demonstrated by MRI, are located in the basal ganglia and/or internal capsule. The prognosis is invariably good, with complete or near-complete recovery. Several publications have emphasized the probable importance of varicella virus in the cause of idiopathic stroke, but the report from Belgium may be the first case-control study demonstrating a significant temporal relationship between idiopathic arterial ischemic strokes of children and varicella-zoster virus infection.

\section{ANTIEPILEPTIC DRUGS}

\section{TOPIRAMATE ADJUNCTIVE THERAPY FOR PARTIAL SEIZURES}

The efficacy and safety of topiramate (TPM) $(6 \mathrm{mg} / \mathrm{kg} /$ day $)$ in children age 2 to 16 years, as adjunctive therapy for uncontrolled partial-onset seizures ( $75 \%$ complex partial), with or without secondarily generalized seizures, were evaluated in a multicenter, randomized, double-blind, placebo-controlled trial, organized by the Topiramate YP Study Group. Comparing 41 TPM-treated with 45 patients receiving placebo, the TPM group showed a greater median percent reduction from baseline in the average monthly partial-onset seizure rate (33\% versus $10 \%$, $\mathrm{p}=0.034$ ), a greater proportion with a $>50 \%$ seizure reduction (39\% versus $20 \%$, $\mathrm{p}=0.08)$ or $>75 \%$ seizure reduction $(17 \%$ versus $2 \%, \mathrm{p}=0.019)$, and better parental 
evaluations of lessened seizure severity $(\mathrm{p}=0.019)$. Adverse effects occurring more frequently among TPM treated than placebo-treated children included emotional lability ( $12 \%$ vs $4 \%$ ), fatigue ( $15 \%$ vs $7 \%)$, impaired attention (12\% vs $2 \%)$, and impaired memory ( $7 \%$ vs $0 \%$ ). Side-effects were not sufficiently severe to require discontinuation of therapy. TPM was considered safe and effective as an adjunctive treatment of partial-onset seizures in children. (Elterman RD, Glauser TA, Wyllie E et al. A double-blind, randomized trial of topiramate as adjunctive therapy for partial-onset seizures in children. Neurology April 1999;52:13381344). (Reprints: Dr Roy Elterman, Dallas Pediatric Neurology Associates, $12801 \mathrm{~N}$ Central Expressway, Plaza 3, Suite 580, Dallas, TX 75230).

COMMENT. In addition to trials in children with partial seizures, topiramate ( $6 \mathrm{mg} / \mathrm{kg} /$ day) as adjunctive therapy has been tested and proven effective and well-tolerated in children and adults with primary generalized tonic-clonic seizures (Biton V, Montouris GD, Ritter F et al. A randomized, placebo-controlled study of topiramate in primary generalized tonic-clonic seizures. Neurology April 1999;52:1330-1337).

\section{VIGABATRIN vs CARBAMAZEPINE IN PARTIAL SEIZURES}

The efficacy and tolerability of vigabatrin (VGB) and carbamazepine (CBZ) monotherapy were compared in an open long-term study in newly diagnosed partial seizures in children treated at the Infantile Neuropsychiatric Division of the Regional Pediatric Hospital, Ancona, Italy. VGB, 50-60 mg/kg/day. in 38 patients or CBZ, $15-20 \mathrm{mg} / \mathrm{kg} /$ day, in 32 patients were split into twice-a-day doses given for a 2-year follow-up period. Clinical efficacy of VGB was similar to that of CBZ ( $76 \%$ vs $78 \%$ benefited, respectively). Relapses occurred in $24 \%$ of the VGB group and $22 \%$ of the CBZ-treated patients.

VGB adverse effects included abnormal weight gain of $10-20 \%$ in $10(26 \%)$ patients within the first 3 months of the study, irritability/excitability and antisocial behavior in $6(16 \%)$, and asymptomatic visual field constriction in an unspecified number. The most significant and troublesome CBZ adverse effect was a generalized urticarial rash within the first 10 days of therapy in $6(19 \%)$ patients, associated with high fever and severe leukopenia in one. Excessive sedation occurred in 6 (19\%). Effects on cognitive function were not addressed. (Zamponi N, Cardinali C. Open comparative long-term study of vigabatrin vs carbamazepine in newly diagnosed partial seizures in children. Arch Neurol May 1999;56:605-607). (Reprints: Nelia Zamponi MD, Department of Pediatric Neurology, "G Salesi" Children's Hospital, Via Corridoni, 11, 60 123, Ancona, Italy).

COMMENT. The clinical efficacy of vigabatrin and carbamazepine monotherapy was comparable in an open long-term trial in children with newly diagnosed partial seizures. The high incidence of skin rash within 10 days of initiating CBZ therapy is a concern, and the role of VGB as an alternative treatment of childhood partial epilepsies should be studied further. The value of VGB as therapy for infantile spasms has been established and reported previously.

VGB response rate and tolerability were superior to other new antiepileptic drugs (gabapentin, lamotrigine, tiagabine) in a meta-analysis comparison of key clinical trials (Cramer JA, Fisher R, Ben-Menachem E, French J, Mattson RH. New antiepileptic drugs: comparison of key clinical trials. Epilepsia May 1999;40:590$600)$. Drawbacks to the use of these data included significant differences among trials in reports of placebo response; lower than average response $(6 \%)$ in tiagabine trials, and higher than average response $(14 \%)$ in topiramate trials. 